\title{
Dynamics of a hole in the $t-J$ model with local disorder: Exact results for high dimensions
}

\author{
Rainer Strack and Dieter Vollhardt \\ Institut für Theoretische Physik C, Technische Hochschule Aachen, D-5100 Aachen, Federal Republic of Germany
}

(Received 22 June 1992)

\begin{abstract}
We investigate the $t-J$ model including local disorder for a hole in an antiferromagnetic spin background on a hypercubic lattice in $d$ dimensions. We show that the well-known approximation of a linear potential, caused by the Ising term of the spin-spin interaction, is correct up to order $1 / d^{2}$ and hence becomes exact in $d=\infty$. We also investigate the influence of disorder and compute exactly the Green function and the density of states for a hole in an antiferromagnetic spin background for arbitrary disorder and $J$ in the limit $d \rightarrow \infty$. Moreover, for finite $J$ and disorder we determine the dynamical conductivity in high dimensions exactly. Remarkable structure both in the density of states and in the dynamical conductivity is found. Finally, we systematically derive the retraceable path approximation by Brinkman and Rice via a self-consistent $1 / d$ expansion, which is correct up to order $1 / d^{4}$.
\end{abstract}

\section{INTRODUCTION}

The investigation of single-particle excitations in magnetic insulators has recently received renewed attention due to the interest in the physical properties of high- $T_{c}$ superconducting materials close to $T_{c}$. This problem involves strongly correlated electrons on a lattice ${ }^{1}$ and a low density of doped holes $\delta$. In the language of the Hubbard model ${ }^{2}$ with strong on-site repulsion $U$ this corresponds to vacancies, i.e., "holes," in an antiferromagnetic background $\left(t-J\right.$ model $\left.^{3-6}\right)$. If, in the limit $\delta<<1$, one neglects the correlations between holes, one arrives at the problem of a single hole in an antiferromagnetic spin background, whose dynamics is described by the $t-J$ model. In many instances the materials under investigation are also disordered to a certain degree. One should expect that the presence of disorder will have a strong influence on this dynamics. It is therefore desirable to include the effect of disorder in the problem under investigation. An understanding of the physics of a single hole in such a disordered, correlated system is not only important for high- $T_{c}$ superconducting materials ${ }^{7}$ but also for magnetic, disordered systems, as, for example, $\mathrm{V}_{1-x} \mathrm{Nb}_{x} \mathrm{O}_{2}{ }^{8}$

Following the work of Nagaoka, ${ }^{9}$ the motion of a hole in the infinite- $U$ Hubbard model was studied in detail by Brinkman and Rice. ${ }^{10}$ They obtained the density of states and the conductivity by means of the so-called retraceable path approximation. This approximation was then also used for the calculation of the dynamical conductivity by Rice and Zhang, ${ }^{11}$ and of the electrical resistivity, thermal conductivity, thermopower, and specific heat by Oguri and Maekawa. ${ }^{12}$ Recently, Metzner, Schmit, and Vollhardt ${ }^{13}$ showed that the retraceable path approximation becomes exact for the Neel background in high dimensions $d$. Moreover, they calculated the spectral function and the dynamical conductivity for antiferromagnetic, ferromagnetic, and random spin backgrounds exactly in $d=\infty$ and derived a self-consistent approximation for these quantities in finite dimensions, in which sum rules are always satisfied.

The influence of finite antiferromagnetic exchange coupling $J$ on the hole motion $^{4}$ has been investigated by several groups. Schmitt-Rink, Varma, and Ruckenstein ${ }^{14}$ and $\mathrm{Kane}$, Lee, and Read ${ }^{15}$ approximated the $t-J$ model at low doping by an effective Hamiltonian and calculated the spectral function from there; a detailed numerical analysis, including vertex corrections, was performed by Liu and Manousakis. ${ }^{16}$ Using the concept of strings generated by the motion of the vacancy, Shraiman and Siggia, ${ }^{17}$ Eder and Becker, ${ }^{18}$ and Mohan ${ }^{19}$ investigated the influence of the Ising term on the hole motion. Gros and Johnson $^{20}$ suggested a Green-function approach starting from a mean-field Ising interaction. Extensive numerical results were obtained by Dagotto et al., ${ }^{21}$ Szczepanski et $a l .,^{22}$ and Inoue and Maekawa. ${ }^{23}$

In the present work we investigate the dynamics of a hole in a Néel state by the $t-J$ model with local disorder in the limit of high dimensions $d$. The density of states and the dynamical conductivity are calculated exactly. These results may be used as a mean-field approximation scheme $\mathrm{e}^{24}$ for results in finite dimensions. By including $1 / d$ corrections our theory can be systematically improved.

The paper is structured as follows. In Sec. II, we introduce the Hamiltonian. We determine the contributions to the hole motion in high dimensions and clarify the influence of spin fluctuations. In Sec. III, the validity of a linear potential due to the Ising interaction is discussed. Moreover, we calculate the Green function exactly in $d=\infty$ for arbitrary $J$. Our results are compared with a continuum approximation. In Sec. IV, the Green function and the density of states and in Sec. $V$ the dynamical conductivity are calculated exactly for arbitrary $J$ and disorder. In Sec. VI, we discuss $1 / d$ corrections to our results for $J=0$ in the absence of disorder, and we derive the retraceable path approximation of Brinkman and Rice $^{10}$ by a self-consistent $1 / d$ correction to our $d=\infty$ result. The summary in Sec. VII concludes the presentation. 


\section{THE $t-J$ MODEL WITH LOCAL DISORDER}

The $t-J$ model $^{3-6}$ is defined on the subspace of zero double occupancy of electrons and consists of a hopping term and a nearest-neighbor spin-spin interaction. We extend this Hamiltonian by adding a term describing local disorder

$$
\hat{H}=\hat{H}_{t}+\hat{H}_{J}+\hat{H}_{v}
$$

with

$$
\begin{aligned}
\hat{H}_{t} & =-t \sum_{\langle\mathrm{ij}\rangle, \sigma}\left(1-\hat{n}_{\mathrm{i}-\sigma}\right) \hat{c}_{\mathrm{i} \sigma}^{\dagger} \hat{c}_{\mathrm{j} \sigma}\left(1-\hat{n}_{\mathrm{j}-\sigma}\right), \\
\hat{H}_{J} & =\hat{H}_{J_{z}}+\hat{H}_{J_{1}} \\
& =\sum_{\langle\mathrm{ij}\rangle}\left[J_{z} \hat{S}_{\mathrm{i}}^{z} \hat{S}_{\mathrm{j}}^{z}+J_{1}\left(\hat{S}_{\mathrm{i}}^{x} \hat{S}_{\mathrm{j}}^{x}+\hat{S}_{\mathrm{i}}^{y} \hat{S}_{\mathrm{j}}^{y}\right)\right], \\
\hat{H}_{v} & =\sum_{\mathrm{i}, \sigma} v_{\mathrm{i}} \hat{n}_{\mathrm{i} \sigma} .
\end{aligned}
$$

Here, $\widehat{c}_{\mathrm{i} \sigma}^{\dagger}\left(\widehat{c}_{\mathrm{i} \sigma}\right)$ creates (annihilates) a particle with spin $\sigma$ on site i. The corresponding number operator is $\hat{n}_{\mathrm{i} \sigma}=\hat{c}_{\mathrm{i} \sigma}^{\dagger} \widehat{c}_{\mathrm{i} \sigma}$. The spin operator is $\widehat{\mathbf{S}}_{\mathrm{i}}$. The index $\langle\mathrm{ij}\rangle$ stands for summation over the nearest-neighbor pairs. The parameter $t$ is the hopping integral, $J_{z}=J_{\perp}=J$ determines the strength of the spin-spin interaction, and $v_{\mathrm{i}}$ characterizes the local disorder and is a stochastic variable. The exchange constant is given by $J=4 t^{2} / U$ when derived from the Hubbard model for large on-site interaction $U$. The Hamiltonian $\hat{H}_{J_{1}}$ describes spin-flip processes. We refer to this model as the $t-J-v_{\mathrm{i}}$ model.

The local Green function for a state $|\mathbf{i}\rangle\rangle$, denoting the Néel state with a hole on site $\mathbf{i}$, is defined as

$$
G_{\mathrm{ii}}(\omega)=\left\langle\mathrm{i} N\left|\frac{1}{\omega-\hat{H}}\right| \mathrm{i} N\right\rangle .
$$

The density of states is then determined by

$$
D(\omega)=\frac{1}{\pi L} \sum_{\mathrm{i}} \operatorname{Im} G_{\mathrm{ii}}\left(\omega-i 0^{+}\right),
$$

where $L$ is the number of lattice sites. For $v_{\mathrm{i}} \neq 0$ one has to average these quantities with respect to the disorder distribution function.

In the case of a single hole moving in a spin background $\left(J=0, v_{\mathrm{i}}=0\right), \mathrm{Nagaoka}^{9}$ and Brinkman and Rice $^{10}$ developed a path formalism to calculate $G_{\mathrm{ii}}$. By expanding $1 /\left(\omega-\hat{H}_{t}\right)$ in a power series in $\left(\hat{H}_{t} / \omega\right)^{n}$ the local Green function can be written as

$$
G_{\mathrm{ii}}(\omega)=1 / \omega\left(1+\sum_{n=2}^{\infty} A_{\mathrm{in}}(-t / \omega)^{n}\right) .
$$

Here the amplitude $A_{\text {in }}$ is the number of distinct paths by which a hole may start at site $\mathrm{i}$ and return to $\mathrm{i}$ after $n$ steps without changing the original spin configuration ("background-restoring paths"). Although this is a seemingly simple problem it is not possible to determine $G_{\text {ii }}$ exactly (even for $J=0, v_{\mathrm{i}}=0$ ) in dimensions $d=2,3$. Only in $d=1$ (Ref. 10) and in $d=\infty$ (Ref. 13) is an exact calculation of the physical properties possible. In the limit $d \rightarrow \infty$, which was recently introduced for lattice models with itinerant degrees of freedom, ${ }^{25}$ substantial technical simplifications occur, while at the same time correlations remain nontrivial. ${ }^{26}$ The $d=\infty$ results may be systematically improved by taking $1 / d$ corrections into account. To obtain a nontrivial theory in the limit $d \rightarrow \infty$ one has to scale the relevant parameters in such a way that the expectation values of $\hat{H}_{t}, \hat{H}_{J}$ remain infinite, i.e.,

$$
\begin{aligned}
& t=\frac{t^{*}}{\sqrt{Z}}, \quad t^{*}=\text { const } \\
& J=\frac{J^{*}}{Z}, \quad J^{*}=\text { const }
\end{aligned}
$$

where $Z=2 d$ is the coordination number on a hypercubical lattice, i.e., the number of nearest neighbors.

In the present problem the essential simplification of the limit $d \rightarrow \infty$ is the suppression of certain paths which only contribute to the Green function in finite dimensions. ${ }^{13}$ This is exemplified in Fig. 1 for $J^{*}=0, v_{\mathrm{i}}=0$. The lowest-order contribution to the Green function due to $\hat{H}_{t}$ is given by $\left(1 / \omega^{3}\right)\left\langle\mathrm{i} N\left|\hat{H}_{t}^{2}\right| \mathrm{i} N\right\rangle$. The corresponding closed path, described by two hopping processes (from site $i$ to a nearest-neighbor site and again back to $i$ ), is shown in Fig. 1(a); it provides a factor $t^{2} \sim 1 / d$, while the number of possible embeddings of this path on the lattice is proportional to $d$. Hence, the product is of order unity and the path is seen to contribute in $d=\infty$. The path in Fig. 1(b) (three loops around the same plaquette) consists of 12 hopping processes which provide a factor $t^{12} \sim 1 / d^{6}$. The number of embeddings of this path is proportional to $d^{2}$. Hence, this path is proportional to $1 / d^{4}$ and obviously vanishes for $d \rightarrow \infty$. Indeed, Metzner, Schmit, and Vollhardt ${ }^{13}$ showed that in $d=\infty$ only those paths of a hole contribute to $G_{\mathrm{ii}}(\omega)$ which consist of loops on which a hole circulates only once ("loop trees"). Loop trees may be divided into two classes: one consisting of paths without loops ("retraceable paths"10) and those containing loops. Since in a Néel state a hole has to circulate three times around the simplest loop [a plaquette, see Fig. 1(b)] to restore the original configuration, loops do not contribute at all in this case. Hence, for a Néel state in $d=\infty$ only retraceable paths remain, and the retraceable path approximation of Brinkman and Rice ${ }^{10}$ becomes exact. ${ }^{13}$

The second simplification of high dimensions concerns the spin-flip term $\hat{H}_{J_{1}}$. We consider only such contributions of $\hat{H}_{J_{1}}$ which influence the hole motion directly. To show that this term does not contribute in $d=\infty$ let us consider the following example: The lowest contribution

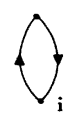

(a)

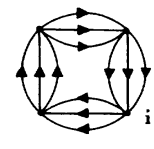

(b)
FIG. 1. Closed paths of a hole in an antiferromagnetic spin background: (a) two hopping processes, (b) 12 hopping processes. 
to the local Green function due to $\hat{H}_{J_{1}}$ is given by $\left(1 / \omega^{6}\right)\left\langle\mathrm{i} N\left|\hat{H}_{J_{\perp}} \hat{H}_{t}^{4}\right| \mathbf{i} N\right\rangle$. After the hole has hopped around a plaquette due to $\hat{H}_{t}^{4}$, two spins are disarranged. By application of $\hat{H}_{J_{\perp}}$ on this state the two disarranged spins are flipped back and we again obtain our starting state $|\mathbf{i} N\rangle$. The path around the plaquette consists of four hopping processes $\left(\sim 1 / d^{2}\right)$, the number of embeddings of the path is proportional to $d^{2}$, and the application of $\hat{H}_{J_{1}}$ provides an additional factor $1 / d$. Hence, this lowest contribution is of order $1 / d$. For a hole moving in an antiferromagnetic spin background in $d=\infty$ spin-flip processes due to $\hat{H}_{J_{\perp}}$ are hence seen to be unimportant, i.e.,

$$
\frac{J^{*}}{2 d} \sum_{\langle\mathrm{ij}\rangle} \widehat{\mathrm{S}}_{\mathrm{i}} \widehat{\mathrm{S}}_{\mathrm{j}} \rightarrow \frac{J^{*}}{2 d} \sum_{\langle\mathrm{ij}\rangle} \hat{S}_{\mathrm{i}}^{z} \hat{S}_{\mathrm{j}}^{z}
$$

where $\hat{S}_{\mathrm{i}}^{z}=\frac{1}{2}\left(\hat{n}_{\mathrm{i} \uparrow}-\hat{n}_{\mathrm{i} \downarrow}\right)$. The ground state of $\hat{H}_{J}$ in $d=\infty$ is the Néel state. ${ }^{27}$ This state is our starting point for the calculation of the hole dynamics in the $t-J-v_{\mathrm{i}} \bmod -$ el.

\section{GREEN FUNCTION FOR THE $t$-J MODEL}

The hole moves into the lattice by the repeated application of $\hat{H}_{t}$ on $|\mathrm{i} N\rangle$, leading to a string of overturned spins. ${ }^{17}$ The Hamiltonian $\hat{H}_{J_{z}}$, applied on a string state, measures the number of overturned spins; its energy increases linearly with the length of the path. Hence, the hole feels a linear potential caused by $\hat{H}_{J_{z}}$. However, this picture is only correct for a path which does not intersect or touch itself as already pointed out by Bulaevskii, Nagaev, and Khomskii. ${ }^{4}$ A self-intersection is depicted in Fig. 2(a): the hole starts at site i, makes one loop, and then retraces to $\mathrm{i}$ (this path is of order $1 / d^{2}$ ). A path which touches itself is shown in Fig. 2(b) (here "touching" means that $\mathbf{j}$ has to be at a nearest-neighbor position to $i)$ : the correction to the linear potential is of order $1 / d^{2}\left(1 / d\right.$ due to $\hat{H}_{t}$ and $1 / d$ due to $\left.\hat{H}_{J_{z}}\right)$. Hence, the approximation of a linear potential, which is frequently used (see, e.g., Ref. 17) and whose validity is not a priori clear, is actually found to be correct up to order $1 / d^{2}$ in high dimensions. Hence, it is indeed a reasonable approximation in $d=2,3$. In particular, in $d=\infty$ the approximation becomes exact. To determine the precise form of this linear potential we define lattice sites $\mathrm{i}+\boldsymbol{\delta}_{n}$, where $\delta_{n}=\tau_{1}+\cdots+\tau_{n}\left(\tau_{n} \neq-\tau_{n-1}\right)$ and $\tau_{n}$ is a unit

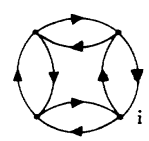

(a)

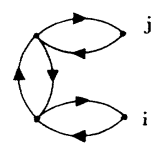

(b)
FIG. 2. Path which (a) intersects at site $i$ and (b) touches at nearest-neighbor positions $\mathbf{i}, \mathbf{j}$. vector on the lattice. After the hole hopped from site $\mathrm{i}$ to $\mathrm{i}+\boldsymbol{\delta}_{n}$ in $n$ steps, it hence experiences a linear potential given by

$$
\widetilde{V}_{n}=n \frac{J^{*}}{2}
$$

in $d=\infty$. Note, that in this limit a path naturally does not intersect or touch itself. We choose the energy of the Néel state with one hole as the zero of energy.

In order to calculate the Green function exactly for the hole in the $t$ - $J$ model we present a diagrammatic representation which is in some sense similar to the locator formalism. ${ }^{28}$ We define a self-energy $\sigma_{\mathrm{i}}$ by

$$
G_{\mathrm{ii}}(\omega)=\frac{1}{\omega\left[1-\sigma_{\mathrm{i}}(\omega)\right]}=\frac{1}{\omega} \sum_{n=1}^{\infty}\left[\sigma_{\mathrm{i}}(\omega)\right]^{n}
$$

The self-energy is given by a sum over paths which start and end at $|\mathbf{i} N\rangle$ without passing $|\mathbf{i} N\rangle$ on the way (a similar self-energy was introduced by Brinkman and Rice ${ }^{10}$ and by Metzner, Schmit, and Vollhardt ${ }^{13}$ ). Equation (8) can be expressed diagrammatically. Diagrammatic elements are shown in Fig. 3(a), where $g_{i}^{0}=1 / \omega$ is the bare Green function for $\hat{H}_{t}=0$. In Fig. 4(a), the diagrammatic representation of Eq. (8) is depicted. In order to determine $\sigma_{\mathrm{i}}$ we have to introduce additional diagrammatic elements [see Fig. 3(b)]. Here $g_{\mathbf{i}+\delta_{n}}$ are auxiliary Green functions (with $\delta_{n}$ defined earlier), $\sigma_{i+\delta_{n}}$ are auxiliary self-energies, $g_{\mathrm{i}+\delta_{n}}^{0}=1 /\left(\omega-\widetilde{V}_{n}\right)$ are the bare Green functions $\left(\hat{H}_{t}=0\right)$ for the string states, and $t$ is the hopping amplitude. The self-energy $\sigma_{i}$ is given by a nearestneighbor hopping from the bare vertex $g_{i}^{0}$ to a dressed vertex $g_{\mathrm{i}+\tau_{1}}$ [see Fig. 4(b)]. ${ }^{29}$ The Green function $g_{\mathbf{i}+\tau_{1}}$ may be expressed by the self-energy $\sigma_{i+\tau_{1}}$ which depends on additional Green functions $g_{\mathrm{i}+\delta_{2}}$ and so on. This general dependence is depicted in Fig. 5 where

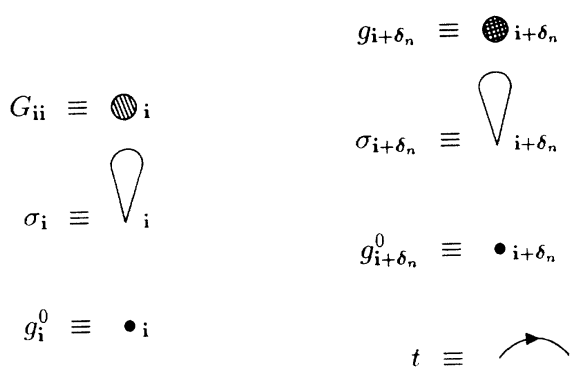

(a)

(b)

FIG. 3. Diagrammatic elements determining (a) the local Green function $G_{\mathrm{ij}}(\omega)$ and (b) the self-energy $\sigma_{\mathrm{i}}(\omega)$. 


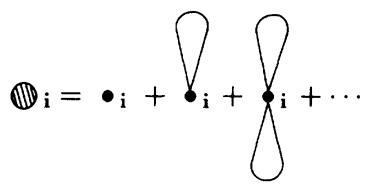

(a)

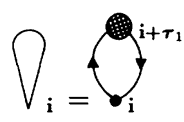

(b)
FIG. 4. Diagrammatic representation (a) of the local Green function $G_{\mathrm{ii}}(\omega)$ and (b) of the self-energy $\sigma_{\mathrm{i}}(\omega)$.

$n=1,2,3, \ldots$. From this diagrammatic analysis we obtain the following set of equations:

$G_{\mathrm{ii}}=\frac{g_{\mathrm{i}}^{0}}{1-\sigma_{\mathrm{i}}}$, $\sigma_{\mathrm{i}}=t^{2} g_{\mathrm{i}}^{0} \sum_{\tau_{1}} g_{\mathrm{i}+\tau_{1}}$,
$g_{\mathrm{i}+\delta_{n}}=\frac{g_{\mathrm{i}+\delta_{n}}^{0}}{1-\sigma_{\mathrm{i}+\delta_{n}}}, \quad n=1,2,3, \ldots$,

$\sigma_{\mathbf{i}+\delta_{n}}=t^{2} g_{\mathbf{i}+\delta_{n}}^{0} \sum_{\tau_{n+1}}^{\prime} g_{\mathrm{i}+\delta_{n+1}}, \quad n=1,2,3, \ldots$

Here the summation with a prime is restricted to $\tau_{n+1} \neq-\tau_{n}$ due to the definition of the self-energies [in $d=\infty$ this restriction is unimportant $(\sim 1 / d)]$. Insertion of the recursion relations into the equation for $G_{\text {ii }}$ yields the following continued fraction

$$
G_{\mathrm{ii}}(\omega)=\frac{1}{\omega-t^{* 2} \frac{1}{\omega-\frac{J^{*}}{2}-t^{* 2} \frac{1}{\omega-2 \frac{J^{*}}{2}-t^{* 2} \frac{1}{\omega-3 \frac{J^{*}}{2}-t^{* 2} \frac{1}{\ddots}}}}}
$$

which may be written as a self-consistent equation for $G_{\mathrm{ii}}$

$$
G_{\mathrm{ii}}(\omega)=\frac{1}{\omega-t^{* 2} G_{\mathrm{ii}}\left(\omega-J^{*} / 2\right)} .
$$

This equation was already obtained by Kane, Lee, and $\operatorname{Read}^{15}$ on the basis of their approximation scheme. Here, it is derived as an exact result in high dimensions. For $J^{*}=0$ we obtain the well-known result

$$
G_{\mathrm{ii}}(\omega)=\frac{\omega}{2 t^{* 2}}\left(1-\sqrt{1-4 t^{* 2} / \omega^{2}}\right),
$$

which was already derived by Metzner, Schmit, and Vollhardt. ${ }^{13}$ It yields a semielliptic density of states with a square-root singularity at the band edges (see Fig. 6). Nondiagonal Green functions are equal to zero due to the antiferromagnetic spin background and hence the spectral function is $\mathbf{k}$ independent. This behavior is also valid in the case of finite $J^{*}$ and $v_{\mathrm{i}}$. For $J^{*} \neq 0$ the continued fraction is equal to a fraction of Bessel functions of the first kind ${ }^{30}$

$$
G_{\mathrm{ii}}(\omega)=-\frac{1}{t^{*}} \frac{\partial_{v}(x)}{\partial_{v-1}(x)}
$$

$\operatorname{li}_{i+\delta_{n}}=\bullet_{i+\delta_{n}}+\oint_{i+\delta_{n}}+\oint^{i+\delta_{n}}+\cdots$

(a)

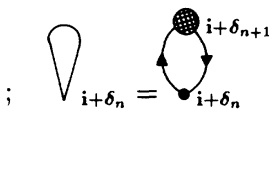

(b)
FIG. 5. Diagrammatic representation (a) of the auxiliary Green functions $g_{\mathbf{i}+\delta_{n}}$ in terms of auxiliary self-energies $\sigma_{\mathbf{i}}+\delta_{n}$ and (b) $\sigma_{\mathrm{i}+\delta_{n}}$ in terms of $g_{\mathrm{i}+\delta_{n+1}}$. with $v=-2 \omega / J^{*}$ and $x=4 t^{*} / J^{*}$. An approximate result for $d=2$ with a similar form was obtained by Mohan. ${ }^{19}$ The Green function may be written as ${ }^{31}$

$$
G_{\mathrm{ii}}(\omega)=\frac{1}{t^{*}} \sum_{n=1}^{\infty}\left[\frac{1}{x-j_{v-1, n}}+\frac{1}{x+j_{v-1, n}}\right],
$$

where $j_{v-1, n}$ are the zeros of $x^{v-1} \partial_{v-1}(x)$. The poles of $G_{\mathrm{ii}}$ are determined by $x \pm j_{v-1, n}=0$. For large $v$ we can solve this equation exactly. The position of the poles near the lower band edge are given by

$$
\omega_{n}=-2 t^{*}-\frac{J^{*}}{2}-a_{n} t^{*}\left[\frac{J^{*}}{2 t^{*}}\right]^{2 / 3},
$$

where $a_{n}$ are the zeros of the Airy function $\operatorname{Ai}(x){ }^{30}$ The

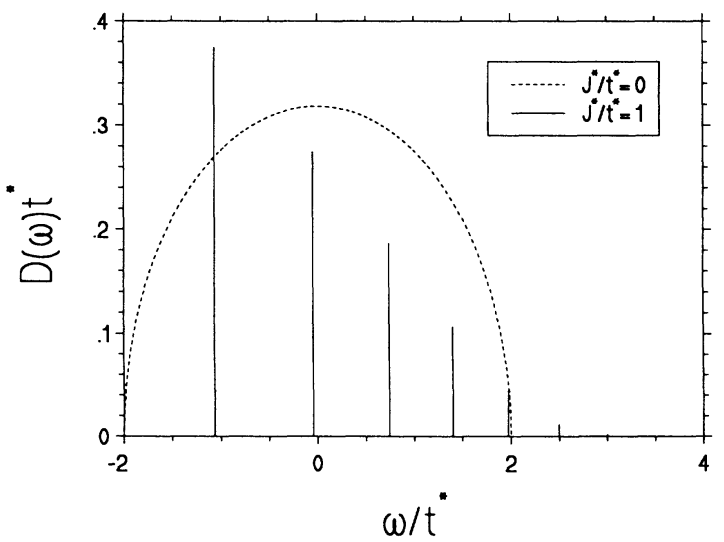

FIG. 6. Density of states for a hole in the $t-J$ model for zero and finite exchange coupling $J^{*}$ in $d=\infty$. 
ground-state energy is obtained for $n=1\left(a_{1}=-2.338\right)$. The $\left[\mathrm{J}^{*} / 2 t^{*}\right]^{2 / 3}$ dependence was already predicted by a continuum approximation, ${ }^{4}$ starting from the threedimensional Schrödinger equation

$$
-t^{*} \Delta \Psi+\frac{J^{*}}{2} r \Psi=\left(E+2 t^{*}+\frac{J^{*}}{2}\right) \Psi .
$$

The term $2 t^{*}+J^{*} / 2$ is due to the scale of the energy. The eigenvalues for angular momentum $l=0$ may be written as

$$
E_{n}=-2 t^{*}-\frac{J^{*}}{2}-a_{n} t^{*}\left[\frac{J^{*}}{2 t^{*}}\right]^{2 / 3}
$$

where $a_{n}$ are the zeros of the Airy function. The continuum approximation is seen to yield exactly the same result as the $d=\infty$ approximation (for small $J^{*}$ and near the bottom of the spectrum). Hence, for large $-\omega / J^{*}$ the continuum approximation and $d=\infty$ are equivalent (at least on the one-particle level). In Fig. 6, we show the density of states for a finite $J^{*}$ as obtained from (10). It is given by a series of $\delta$ peaks; their weight is characterized by their height. In finite dimensions one might think that the spin-flip processes created by $\hat{H}_{J_{1}}$ would completely erase the strings and the $\delta$ peaks, respectively. However, Dagotto et $a .^{21}$ showed by an exact diagonalization on a $4 \times 4$ lattice that this is not the case; the same conclusion was reached by Liu and Manousakis. ${ }^{16}$

An alternative way to calculate $G_{\mathrm{ii}}$ exactly in $d=\infty$ is to write the Green function $G_{\mathrm{ii}}$ as

$$
G_{\mathrm{ii}}(\omega)=\left\langle\mathrm{i} N\left|\frac{1}{\omega-\hat{T}}\right| \mathrm{i} N\right\rangle,
$$

where $\widehat{T}$ is given by $\widehat{T}=1 /\left(1-\hat{H}_{J} / \omega\right) \hat{H}_{t}$. The operator $\hat{T}$ describes a kinetic energy with a site-dependent hopping amplitude. Hence, the hopping amplitude $t_{n}$ between sites $\mathbf{i}+\boldsymbol{\delta}_{n-1}$ and $\mathbf{i}+\boldsymbol{\delta}_{n}$ is given by $t_{n}=t /\left[1-n J^{*} /(2 \omega)\right]$. The diagrammatic representation of (18) is exactly the same as above with $g_{\mathrm{i}}^{0}=g_{\mathrm{i}+\delta_{n}}^{0}=1 / \omega$. The result is again the self-consistent equation (11).

\section{GREEN FUNCTION FOR THE $t-J-v_{i}$ MODEL}

The energy $v_{\mathrm{i}}$ is a stochastic variable with a distribution function $P\left(v_{\mathrm{i}}\right)$ (correlations between sites are neglected). Here, $\mathbf{i}$ runs over all lattice sites $\mathbf{R}_{i}$. A physical quantity like the Green function $G_{\mathrm{ii}}$ depends on all $v_{\mathrm{i}}$. The average of a quantity $Y\left(\left\{v_{i}\right\}\right)$ is then obtained by

$$
\begin{aligned}
\bar{Y} & =\langle Y\rangle_{\mathrm{av}} \\
& =\int d v_{\mathrm{R}_{1}} \cdots \int d v_{\mathrm{R}_{L}} Y\left(v_{\mathrm{R}_{1}}, \ldots, v_{\mathrm{R}_{L}}\right) \prod_{k=1}^{L} P\left(v_{\mathbf{R}_{k}}\right) .
\end{aligned}
$$

In the following we will employ a binary alloy distribution

$$
P(v)=c \delta(v-W / 2)+(1-c) \delta(v+W / 2),
$$

a box distribution

$$
P(v)=\frac{1}{W} \Theta\left[\frac{W}{2}-|v|\right],
$$

and a semielliptic distribution

$$
P(v)=\frac{4}{\pi W} \sqrt{1-4 v^{2} / W^{2}} .
$$

Here, $\delta(x)$ is the $\delta$ function and $\Theta(x)$ is the step function. The parameter $W$ determines the width of the distribution and $c$ measures the concentration of the impurities. As in the previous chapter a self-energy is introduced by

$$
G_{\mathrm{ii}}(\omega)=\frac{1}{\left(\omega+v_{\mathrm{i}}\right)\left[1-\sigma_{\mathrm{i}}(\omega)\right]} .
$$

The diagrammatic representation is exactly the same as before. However, due to the additional local potential the bare quantities $g^{0}$ now take the values $g_{\mathrm{i}}^{0}=1 /\left(\omega+v_{\mathrm{i}}\right)$ and $g_{\mathrm{i}+\delta_{n}}^{0}=1 /\left(\omega+v_{\mathrm{i}+\delta_{n}}-\widetilde{V}_{n}\right)$. Equations $(9 \mathrm{a})-(9 \mathrm{~d})$ are also valid in the case of disorder. It remains to explain how the averaging for $G_{\mathrm{ii}}$ is performed. If one inserts the result for $\sigma_{\mathrm{i}}$ into the equation for $G_{\mathrm{ii}}$, one obtains for the averaged Green function

$$
\overline{G_{\mathrm{ii}}}=\left\langle\frac{1}{\omega+v_{\mathrm{i}}-t^{2} \sum_{\tau_{1}} g_{\mathrm{i}+\tau_{1}}}\right\rangle_{\mathrm{av}},
$$

where $g_{\mathrm{i}+\tau_{1}}$ is independent of $v_{\mathrm{i}}$. To determine $\overline{G_{\mathrm{ii}}}$ averages of powers of $(1 / 2 d) \Sigma_{\tau_{1}} g_{i+\tau_{1}}$ have to be calculated. They become uncorrelated in $d=\infty$, i.e.,

$$
\left\langle\left(\frac{1}{2 d} \sum_{\tau_{1}} g_{\mathrm{i}+\tau_{1}}\right)^{2}\right\rangle_{\mathrm{av}}=\left\langle\frac{1}{2 d} \sum_{\tau_{1}}\left\langle g_{\mathrm{i}+\tau_{1}}\right\rangle_{\mathrm{av}}\right)^{2}
$$

This fact has already been observed by Vlaming and Vollhardt. ${ }^{28}$ Hence, $\bar{G}_{\mathrm{ii}}$ may be written as

$$
\overline{G_{\mathrm{ii}}}=\int d v P(v) \frac{1}{\omega+v-\left(t^{* 2} / 2 d\right) \sum_{\tau_{1}} \overline{g_{\mathrm{i}+\tau_{1}}}}
$$

The same averaging procedure is valid also for the other Green functions with the result 


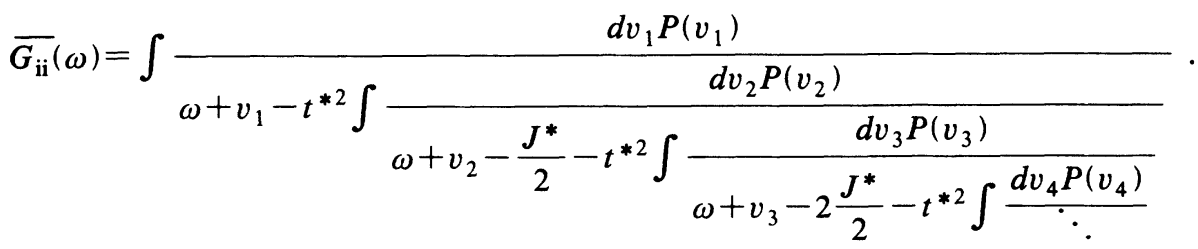

(a)

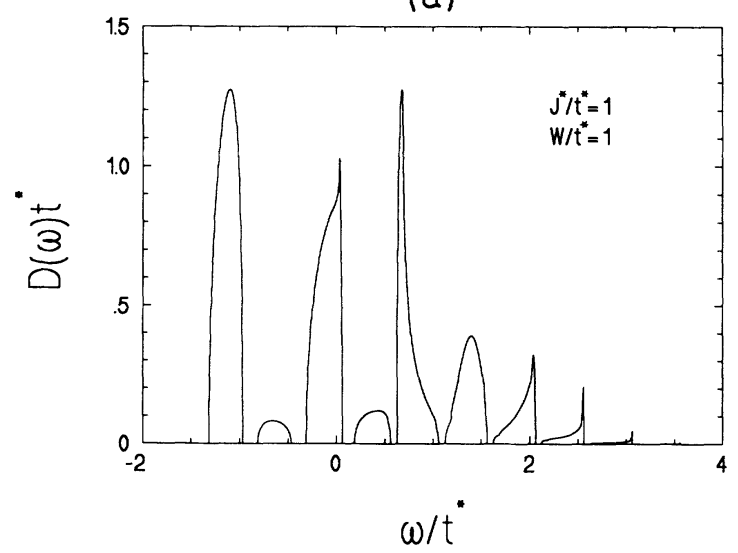

(b)

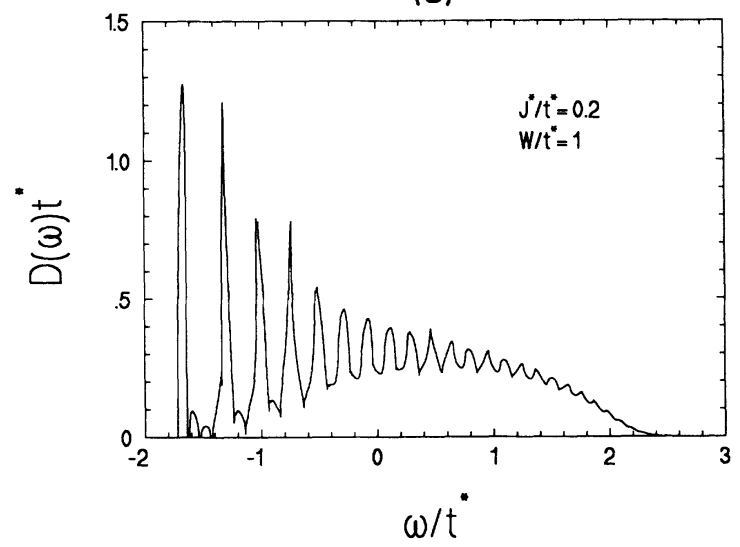

(c)

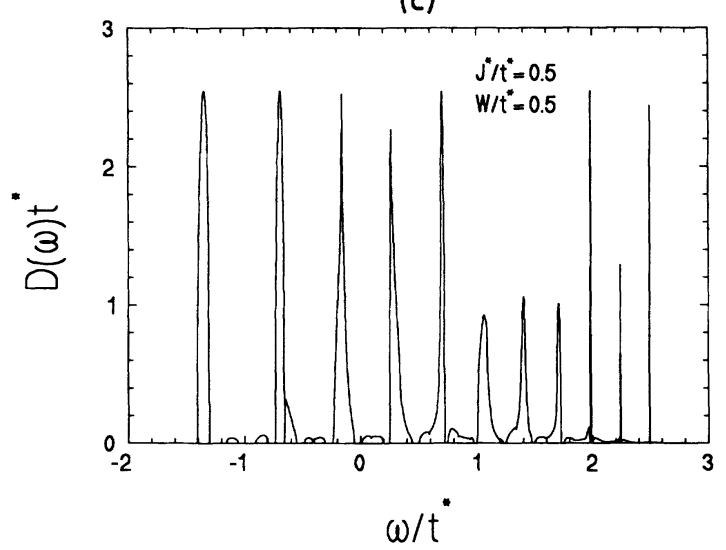

FIG. 7. Density of states for a hole in the $t-J-v_{\mathrm{i}}$ model in $d=\infty$ (semielliptic disorder distribution): (a) $J^{*} / t^{*}=1$, $W / t^{*}=1 ; \quad$ (b) $J^{*} / t^{*}=0.2, \quad W / t^{*}=1 ; \quad$ (c) $J^{*} / t^{*}=0.5$, $W / t^{*}=0.5$.
Again this expression can be written as a self-consistent equation for $\overline{G_{\mathrm{ii}}}$ :

$$
\overline{G_{\mathrm{ii}}}(\omega)=\int d v P(v) \frac{1}{\omega+v-t^{* 2} \overline{G_{\mathrm{ii}}}\left(\omega-J^{*} / 2\right)} .
$$

First we study this relation for $J^{*}=0$. Brouers, Lederer, and Héritier ${ }^{8}$ investigated the case of a binary alloy distribution and obtained a relation for $\overline{G_{i i}}$ which is identical to our result for $Z=\infty$. Their approximation, whose validity could not be assessed before, is seen to become exact in $d=\infty$. Due to the absence of loops, the antiferromagnetic background forces the hole to move on paths which are identical to paths on a Bethe lattice ${ }^{32}$ with a

(a)

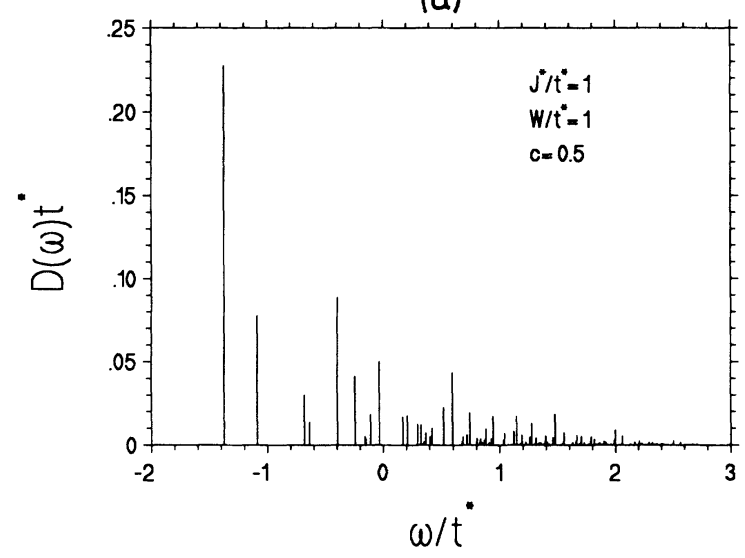

(b)

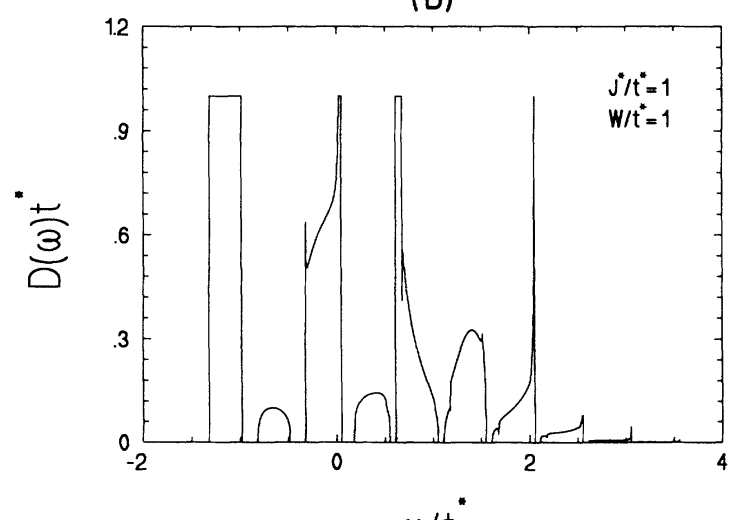

$\omega / t$

FIG. 8. Density of states for a hole in the $t-J-v_{\mathrm{i}}$ model in $d=\infty$ : (a) binary alloy disorder distribution, (b) box disorder distribution. 
coordination number $Z=\infty$. Indeed, for $J^{*}=0,(26)$ is identical to the exact local Green function for the Anderson disorder problem on a $Z=\infty$ Bethe lattice. ${ }^{28}$

For finite $J^{*}$ we may compute the density of states numerically from (26). The recursion relation converges very rapidly. For a semielliptic distribution the original $\delta$ peaks now acquire a finite width (see Fig. 7), and new bands arise. The smaller the width $W$ of the distribution function, the narrower the peaks. A finite width of the peaks is also found if a next-nearest-neighbor hopping $t_{2}^{*}$ ( $t_{2}$ has to be scaled with $1 / Z$ ) instead of disorder is included. $^{33}$ Due to this additional kinetic energy the hole moves on the lattice without destroying the antiferromagnetic spin background. To consider this effect we investigate $\hat{H}_{t}+\hat{H}_{J}+\hat{H}_{t_{2}}$ on a Bethe lattice $(Z=\infty)$ and obtain, for $G_{\mathrm{ii}}$,

$$
\begin{aligned}
G_{\mathrm{ii}}(\omega)= & \frac{\omega-t^{* 2} G_{\mathrm{ii}}\left(\omega-J^{*} / 2\right)}{2 t_{2}^{* 2}} \\
& \times\left[1-\left[1-\frac{4 t_{2}^{* 2}}{\omega-t^{* 2} G_{\mathrm{ii}}\left(\omega-J^{*} / 2\right)}\right]^{1 / 2}\right] .
\end{aligned}
$$

This is exactly the same equation as (26) for a semielliptic distribution and $W^{2}=16 t_{2}^{* 2}$. Consequently, the effect of disorder on $G_{\mathrm{ii}}$ is similar to the effect of an additional kinetic energy that does not destroy the antiferromagnetic spin background. We also investigated the case of a binary alloy distribution and a box distribution (see Fig. 8 ). In the former case the number of new $\delta$ peaks increases rapidly (their weight is characterized by their height). In the latter case the qualitative behavior is the same as for the semielliptic distribution.

\section{DYNAMICAL CONDUCTIVITY FOR THE $t-J-v_{\mathrm{i}}$ MODEL}

The problem of a single hole in a magnetic insulator is of direct physical relevance, since single-particle excitations can actually be studied by spectroscopic methods. In this respect the electrical conductivity provides characteristic information about the physical properties of the solid. Here, we calculate the frequency-dependent conductivity of a hole in the $t-J-v_{\mathrm{i}}$ model for an antiferromagnetic spin background exactly in $d=\infty$. We start from the Kubo formula ${ }^{34}$

$$
\sigma(\omega)=\frac{1}{V} \int_{0}^{\infty} d \tau e^{i \omega \tau} \int_{0}^{\beta} d \lambda\left\langle\widehat{J}_{\alpha}(0) \widehat{J}_{\alpha}(\tau+i \lambda)\right\rangle,
$$

where $V$ is the volume, and $\beta=1 /\left(k_{B} T\right)$ is the inverse temperature. The current operator $\widehat{J}_{\alpha}$ in the direction $\alpha$ ( $\alpha$ is an arbitrary unit vector on the lattice) is given by

$$
\widehat{J}_{\alpha}=\text { ieta } \sum_{\mathbf{j}, \sigma}\left(\widehat{c}_{\mathbf{j}+\alpha \sigma}^{\dagger} \widehat{c}_{\mathbf{j} \sigma}-\hat{c}_{\mathbf{j}-\alpha \sigma}^{\dagger} \widehat{c}_{\mathbf{j} \sigma}\right)
$$

and

$$
\widehat{J}_{\boldsymbol{\alpha}}(\tau)=e^{i \hat{H} \tau} \widehat{J}_{\alpha} e^{-i \hat{H} \tau}
$$

with $a$ as the lattice constant. The thermal average is defined by

$$
\langle\cdots\rangle=\frac{1}{Z} \operatorname{tr}\left(e^{-\beta \hat{H}} \cdots\right),
$$

where the trace runs over all single hole states in a Néel background. The partition function has the form

$$
Z=L \int_{-\infty}^{\infty} d \omega e^{-\beta \omega} D(\omega)
$$

where $D(\omega)$ is the density of states calculated earlier. Following Rice and $\mathrm{Zhang}^{11}$ one can transform the real part of the conductivity into

$\operatorname{Re} \sigma(\omega)=\frac{e^{-\beta \omega}-1}{4 \pi V Z \omega} \int_{-\infty}^{\infty} d \omega_{1} e^{-\beta \omega_{1}} F\left(\omega_{1}, \omega_{1}+\omega\right)$,

where

$F\left(\omega_{1}, \omega_{2}\right)=\sum_{s_{1}, s_{2}= \pm 1} s_{1} s_{2} f\left(\omega_{1}+i s_{1} 0^{+}, \omega_{2}+i s_{2} 0^{+}\right)$

with the definition

$$
f\left(z_{1}, z_{2}\right)=\operatorname{tr}\left(\frac{1}{z_{1}-\hat{H}} \hat{J}_{\alpha} \frac{1}{z_{2}-\hat{H}} \hat{J}_{\alpha}\right) .
$$

These relations are valid in every dimension. Due to the nature of the current operator the conductivity is proportional to $1 / d$ in high dimensions, but $\sigma / t^{2}$ is a finite quantity. In order to obtain an exact result for $\sigma / t^{2}$ in high dimensions we have to expand $1 /(z-\hat{H})$ into a power series as in the case of the Green function. Since nondiagonal Green functions are equal to zero, only the diagram in Fig. 9 remains, ${ }^{13,35}$ where the dashed line represents the hopping caused by the current operator, and the solid circles are full Green functions. In $d=\infty$ both Green functions become uncorrelated, i.e., the averaging procedure can be performed separately with the result

$$
f\left(z_{1}, z_{2}\right)=\frac{e^{2} t^{* 2} a^{2}}{2 d} \sum_{\mathrm{i}}\left[\overline{g_{\mathrm{i}+\alpha}}\left(z_{2}\right)+\overline{g_{\mathrm{i}-\alpha}}\left(z_{2}\right)\right] \overline{G_{\mathrm{ii}}}\left(z_{1}\right) \text {. }
$$

From Eqs. (24) and (25) it follows that

$$
\overline{g_{\mathrm{i}-\alpha}}(\omega)=\overline{g_{\mathrm{i}+\alpha}}(\omega)=\overline{G_{\mathrm{ii}}}\left(\omega-J^{*} / 2\right) .
$$

Hence, the dynamical conductivity is given by

$$
\begin{aligned}
\operatorname{Re} \sigma(\omega)= & \frac{1-e^{-\beta \omega}}{V Z \omega d} e^{2} t^{* 2} a^{2} \pi L \\
& \times \int_{-\infty}^{\infty} d \omega_{1} e^{-\beta \omega_{1}} D\left(\omega_{1}\right) D\left(\omega_{1}+\omega-J^{*} / 2\right),
\end{aligned}
$$

where the density of states was determined earlier. Since

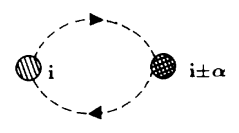

FIG. 9. Diagrammatic representation of the dynamical conductivity in $d=\infty$ in terms of the current operator (dashed line) and the local Green functions $G_{\mathrm{ii}}$ and $g_{\mathrm{i} \pm \alpha}$. 
our result is exact in high dimensions, the sum rule for the conductivity is naturally fulfilled for finite $J^{*}$ and disorder. For finite $J^{*}$ in the absence of disorder the conductivity is characterized by a series of $\delta$ peaks. The general case is shown in Fig. 10 for various values of $J^{*}$ and $W$ in the case of a semielliptic distribution. The $\delta$ peaks are now broadened.

The case $T=0$ is special, namely, the conductivity is given by

$$
\left.\operatorname{Re} \sigma(\omega)\right|_{T=0}=\frac{e^{2} t^{* 2} a^{2} \pi}{V d \omega} D\left(\omega_{\mathrm{low}}+\omega-J^{*} / 2\right)
$$

where $\omega_{\text {low }}$ is the lower band edge of the density of states, i.e., the ground-state energy of the hole (see Fig. 11). We find that the dc conductivity $(T=0)$ is always zero for finite $J^{*}$. For arbitrarily small $J^{*}$ the hole is bound to the origin and thus the dc conductivity drops from infinity for $J^{*}=0$ to zero for $J^{*} \neq 0$. The conductivity and the density of states are hence drastically changed by

(a)

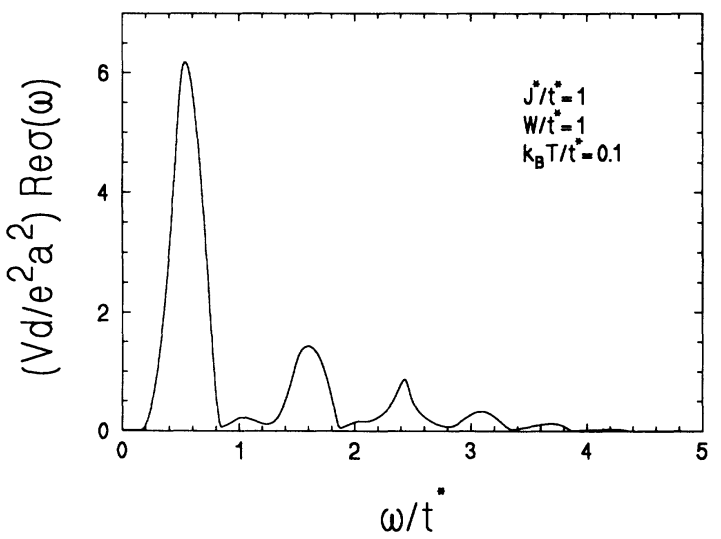

(b)

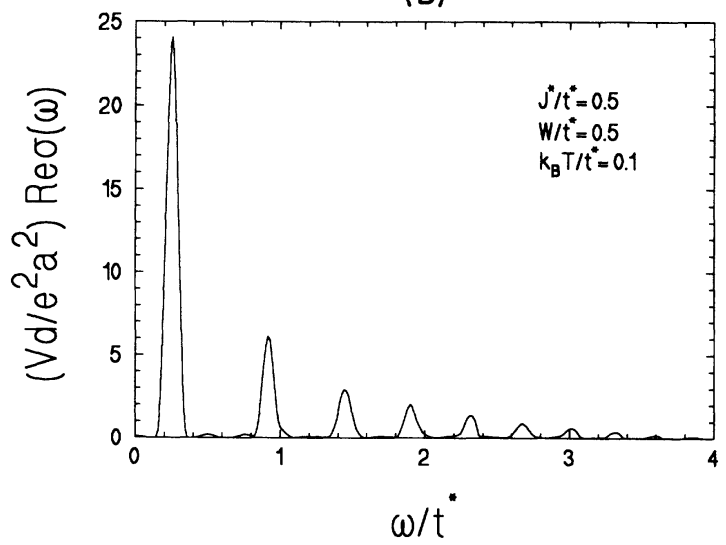

FIG. 10. Dynamical conductivity $\sigma(\omega)$ for a hole in the $t-J-v_{\mathrm{i}}$ model in $d=\infty$ (semielliptic disorder distribution): (a) $J^{*} / t^{*}=1, \quad W / t^{*}=1, \quad k_{B} T / t^{*}=0.1 ; \quad$ (b) $\quad J^{*} / t^{*}=0.5$, $W / t^{*}=0.5, k_{B} T / t^{*}=0.1$.

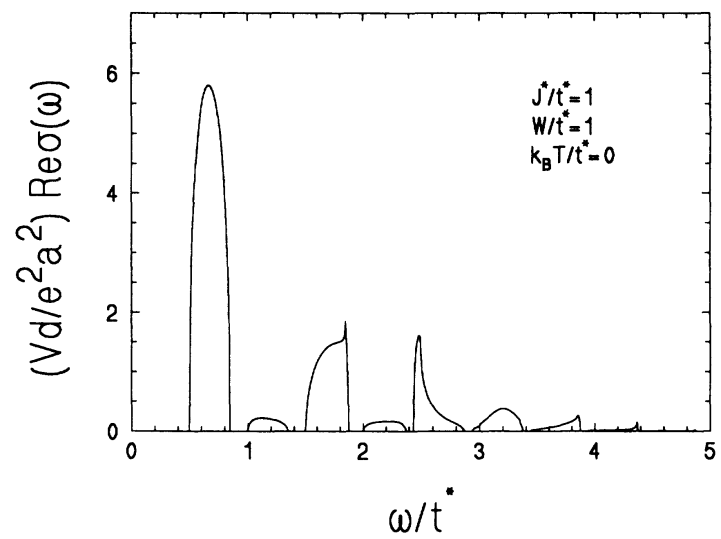

FIG. 11. Dynamical conductivity $\sigma(\omega)$ as in Fig. 10 for $T=0$.

$J^{*}$. Note, that the suppression of $\hat{H}_{J_{1}}$ in high dimensions implies that there is no Drude peak for $v_{i}=0$ in $d=\infty$; of course, in finite dimensions a Drude peak will exist.

In Fig. 12, the temperature dependence of the resistivity is depicted. For increasing temperatures $\rho(T)$ first decreases, then reaches a minimum, and finally increases again slightly. One has to keep in mind that for high temperatures the Néel state will be destroyed and therefore it is not clear how far our results are applied in this limit.

Another interesting fact is the dependence of the dc conductivity on the strength of the disorder as shown in Fig. 13. Disorder is seen to be favorable for the conductivity in a certain disorder interval. As already mentioned in Sec. IV the disorder has some features of an additional kinetic energy and thus this dependence is not surprising. For large $W$ the dc conductivity decreases, as one would expect. We obtain a maximum of $\sigma$ for a certain disorder width. Moreover, the dc conductivity decreases for increasing $J^{*}$.

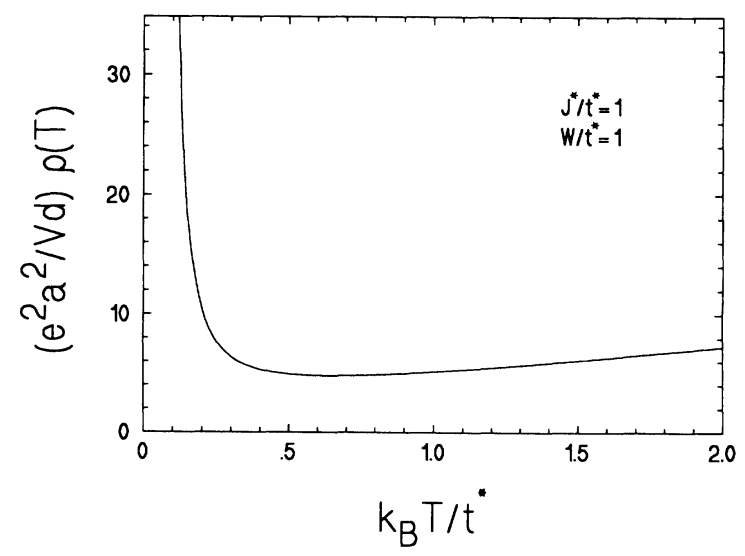

FIG. 12. Resistivity $\rho(T)$ for a hole in the $t-J-v_{\mathrm{i}}$ model in $d=\infty$ (semielliptic disorder distribution). 


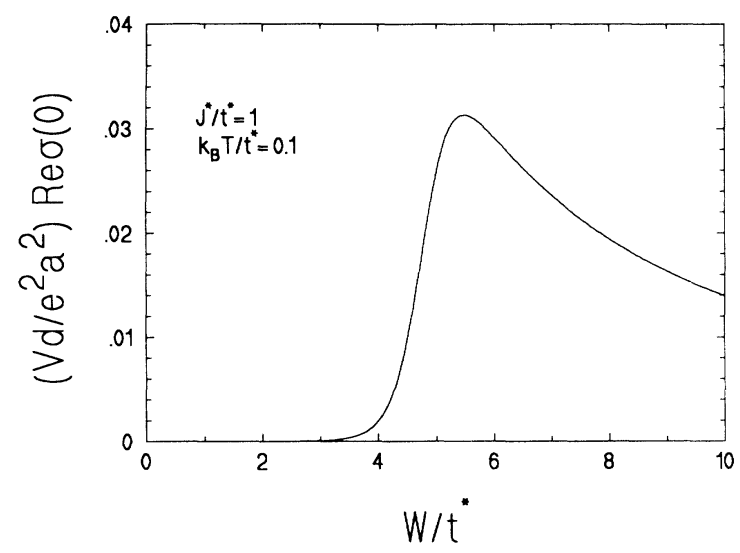

FIG. 13. dc conductivity vs disorder strength $W$ for a hole in the $t-J-v_{\mathrm{i}}$ model in $d=\infty$ (semielliptic disorder distribution).

\section{SELF-CONSISTENT $1 / d$ CORRECTIONS}

The results for $d=\infty$ can be systematically improved by including $1 / d$ corrections. The value of a diagram or a path, respectively, can be exactly determined in terms of an expansion in $1 / d$. The first correction to the $d=\infty$ result for the Green function is given by $G_{\mathrm{ii}}=G_{\mathrm{ii}}^{d=\infty}+1 / d G_{\mathrm{ii}}^{1 / d}$. However, this simple-minded procedure often provides unphysical properties for $G_{\mathrm{ii}}$, e.g., negative density of states. ${ }^{28}$ Hence, one has to include higher orders in $1 / d$ in such a way that the unphysical behavior disappears. One way to do this is to expand the self-energy in terms of $1 / d$ where the Green functions are determined self-consistently. The Green function then includes $1 / d$ contributions to infinite order. A selfconsistent $1 / d$ expansion will now be constructed for $J^{*}=0, v_{\mathrm{i}}=0$. For a $1 / d$ expansion, correct up to order $1 / d$, Eqs. (8) and (9a)-(9d) with $g_{\mathrm{i}}^{0}=g_{\mathrm{i}+\delta_{n}}^{0}=1 / \omega$ may be used. The self-energy $\sigma_{\mathrm{i}}$ is given by $\sigma_{\mathrm{i}}=t^{* 2} /(2 d \omega) \Sigma_{\tau_{1}} g_{\mathrm{i}+\tau_{1}}$. For $\sigma_{\mathrm{i}}$ a correction to order $1 / d$ does not exist (corrections only come in $1 / d^{4}$ ). The self-energy that arises next is

$$
\sigma_{\mathrm{i}+\tau_{1}}=t^{* 2} /(2 d \omega) \sum_{\tau_{2}} g_{\mathrm{i}+\delta_{2}}-t^{* 2} /(2 d \omega) g_{\mathrm{i}}
$$

The first term is the $d=\infty$ result and the second term is the $1 / d$ correction due to the restriction of the summation which excludes the return of a particle (see Sec. II). Consequently, the auxiliary self-energies are given by

$$
\sigma_{\mathrm{i}+\delta_{n}}=t^{* 2} /(2 d \omega) \sum_{\tau_{n+1}} g_{\mathrm{i}+\delta_{n+1}}-t^{* 2} /(2 d \omega) g_{\mathrm{i}+\delta_{n-1}}
$$

leading to a continued fraction

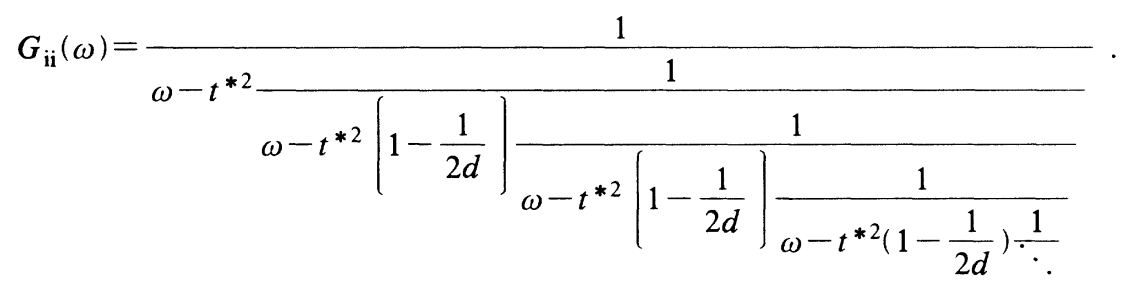

To solve (39) a new quantity $G_{1}(\omega)$ is introduced as

$$
\begin{aligned}
G_{1}(\omega) & =\frac{1}{\omega-t^{* 2}\left(1-\frac{1}{2 d}\right) \frac{1}{\omega-t^{* 2}\left(1-\frac{1}{2 d}\right) \frac{1}{\omega-t^{* 2}\left(1-\frac{1}{2 d}\right) \frac{1}{\ddots}}}} \\
& =\frac{1}{\omega-t^{* 2}(1-1 / 2 d) G_{1}(\omega)} .
\end{aligned}
$$

This yields a quadratic equation for $G_{1}$. Hence, one finally obtains

$G_{\mathrm{ii}}(\omega)=\frac{\omega(1-1 / d)-\sqrt{\omega^{2}-4 t^{* 2}[1-1 /(2 d)]}}{2 t^{* 2}-\omega^{2} / d}$

It should be noted that $(41)$ is identical to the result ob- tained by Brinkman and Rice ${ }^{10}$ by means of the retraceable path approximation in finite dimensions (with $t^{*}=\sqrt{2 d} t$. Hence, we see that the retraceable path approximation is equivalent to a self-consistent $1 / d$ correction to the $d=\infty$ result for a hole moving in an antiferromagnetic spin background. Here, we derived this approximation in a systematic way. Loops enter in order 
$1 / d^{4}$ [see Fig. 1(b)]. Hence, Eq. (41) is valid up to order $1 / d^{4}$.

\section{SUMMARY}

In this paper we investigated the dynamics of a hole in a quantum antiferromagnetic with local disorder. We employed the limit of high dimensions $d$, since it enables us to solve this problem exactly in $d=\infty$. In particular, the effect of finite exchange coupling $J^{*}$ and of the disorder can be treated simultaneously in this approach. These results correspond to a mean-field theory ${ }^{24}$ for finite dimensions $(d=2,3)$. Sum rules for the density of states and the dynamical conductivity are naturally satisfied by our exact results. We showed that the approximation of a linear potential due to $\hat{H}_{J_{z}}$ is valid up to order $1 / d^{2}$. Hence, it can be viewed as a reasonable approximation even in $d=2,3$. In the case of large $-\omega / J^{*}$ and no disorder our results for the low-lying eigenvalues are the same as those obtained from a continuum approximation. These eigenvalues obey a $\left(J^{*} / t^{*}\right)^{2 / 3}$ law, where the prefactor is determined by the zeros of the Airy function. In the case of finite disorder the $\delta$ peaks (due to the linear potential) now acquire a finite width and the density of states has a new structure. Numerical evaluations were obtained for three different types of disorder distribution functions.

For the dynamical conductivity an exact result has been obtained, too, in $d=\infty$. The frequency dependence of $\sigma(\omega)$ is found to have a remarkable structure. The resistivity $\rho(T)$ versus $T$ has a minimum. Moreover, the presence of disorder leads to a new effect, where $\sigma(0)$ increases for increasing disorder in a certain disorder interval. Finally, we systematically included $1 / d$ corrections for $J^{*}=0, v_{\mathrm{i}}=0$. The results of the retraceable path approximation in finite dimensions of Brinkman and Rice ${ }^{10}$ were thereby derived as a self-consistent $1 / d$ correction to the $d=\infty$ result. The calculation of $1 / d$ corrections for finite $J^{*}$, including spin fluctuations, and the consequences of a next-nearest-neighbor hopping are presently under investigation.

\section{ACKNOWLEDGMENTS}

We wish to thank V. Janiš, P. Schmit, G. S. Uhrig, and R. Vlaming for helpful discussions. One of us (R.S.) gratefully acknowledges a scholarship of the Studienstiftung des Deutschen Volkes. This work was supported in part by the Sonderforschungsbereich 341 of the Deutsche Forschungsgemeinschaft.
${ }^{1}$ P. W. Anderson, Science 235, 1196 (1987).

${ }^{2}$ M. C. Gutzwiller, Phys. Rev. Lett. 10, 159 (1963); J. Hubbard, Proc. R. Soc. London, Ser. A 276, 238 (1963); J. Kanamori, Prog. Theor. Phys. 30, 275 (1963).

${ }^{3}$ A. B. Harris and R. V. Lange, Phys. Rev. 157, 295 (1967).

${ }^{4}$ L. N. Bulaevskii, É. L. Nagaev, and D. I. Khomskii, Zh. Eksp. Teor. Fiz. 54, 1562 (1968) [Sov. Phys. JETP 27, 836 (1968)].

${ }^{5}$ K. A. Chao, J. Spałek, and A. M. Oleś, J. Phys. C 10, L271 (1977).

${ }^{6}$ C. Gros, R. Joynt, and T. M. Rice, Phys. Rev. B 36, 381 (1987).

${ }^{7} \mathrm{G}$. A. Thomas, in High Temperature Superconductivity, edited by D. P. Tunstall and W. Barford (Hilger, Bristol, 1991).

${ }^{8} \mathrm{~F}$. Brouers, P. Lederer, and M. Héritier, in Amorphous Magnetism, edited by H. O. Hooper (Plenum, New York, 1973).

${ }^{9}$ Y. Nagaoka, Phys. Rev. 147, 392 (1966).

${ }^{10}$ W. F. Brinkman and T. M. Rice, Phys. Rev. B 2, 1324 (1970).

${ }^{11}$ T. M. Rice and F. C. Zhang, Phys. Rev. B 39, 815 (1989).

${ }^{12}$ A. Oguri and S. Maekawa, Phys. Rev. B 41, 6977 (1990).

${ }^{13}$ W. Metzner, P. Schmit, and D. Vollhardt, Phys. Rev. B 45, 2237 (1992).

${ }^{14}$ S. Schmitt-Rink, C. M. Varma, and A. E. Ruckenstein, Phys. Rev. Lett. 60, 2793 (1988).

${ }^{15}$ C. L. Kane, P. A. Lee, and N. Read, Phys. Rev. B 39, 6880 (1989).

${ }^{16}$ Z. Liu and E. Manousakis, Phys. Rev. B 45, 2425 (1992).

${ }^{17}$ B. I. Shraiman and E. D. Siggia, Phys. Rev. Lett. 60, 740 (1988).

${ }^{18}$ R. Eder and K. W. Becker, Z. Phys. B 78, 219 (1990).

${ }^{19}$ M. M. Mohan, J. Phys.: Condens. Matter 3, 4307 (1991).
${ }^{20}$ C. Gros and M. D. Johnson, Phys. Rev. B 40, 9423 (1989).

${ }^{21}$ E. Dagotto, R. Joynt, A. Moreo, S. Bacci, and E. Gagliano, Phys. Rev. B 41, 9049 (1990).

${ }^{22}$ K. J. v. Szczepanski, P. Horsch, W. Stephan, and M. Ziegler, Phys. Rev. B 41, 2017 (1990).

23J. Inoue and S. Maekawa, J. Phys. Soc. Jpn. 59, 2110 (1990).

${ }^{24}$ P. van Dongen and D. Vollhardt, Phys. Rev. Lett. 65, 1663 (1990).

${ }^{25}$ W. Metzner and D. Vollhardt, Phys. Rev. Lett. 62, 324 (1989).

${ }^{26}$ For reviews, see D. Vollhardt, Physica B 169, 277 (1991); E. Müller-Hartmann, Int. J. Mod. Phys. B 3, 2169 (1989).

${ }^{27}$ T. Kennedy, E. H. Lieb, and B. S. Shastry, Phys. Rev. Lett. 61, 2582 (1988).

${ }^{28}$ R. Vlaming and D. Vollhardt, Phys. Rev. B 45, 4637 (1992).

${ }^{29}$ For $J^{*}=0$ the auxiliary Green function $g_{\mathrm{i}+\delta_{1}}$ is equal to $G_{\mathrm{ii}}$.

${ }^{30}$ M. Abramowitz and I. A. Stegun, Handbook of Mathematical Functions (Dover, New York, 1972).

${ }^{31}$ G. N. Watson, Theory of Bessel Functions (Cambridge University Press, Cambridge, 1958).

${ }^{32}$ E. N. Economou, Green's Functions in Quantum Physics (Springer, Berlin, 1979).

${ }^{33}$ Here we consider a kinetic energy where a $\sigma$ electron can hop to a next-nearest-neighbor site only if the respective nearestneighbor electron has an orientation $(-5)$. Such a term arises in the derivation of the $t-J$ model from the Hubbard model.

${ }^{34}$ See, for example, G. D. Mahan, Many-Particle Physics (Plenum, New York, 1990).

${ }^{35}$ A. Khurana, Phys. Rev. Lett. 64, C1990 (1990). 